\title{
Successful High-Dosage Dexamethasone Treatment of H1N1 Influenza A Pneumonia Complicated with Acute Necrotizing Encephalitis in an HIV-Infected Adult
}

\author{
Kuan-Chih Chen', Chien-Hung Gow ${ }^{2}$, Chia-Jui Yang³, Hou-Tai Chang' \\ ${ }^{1}$ Department of Internal Medicine, Far Eastern Memorial Hospital, New Taipei City, Taiwan \\ ${ }^{2}$ Department of Internal Medicine, Division of Pulmonary Medicine, Far Eastern Memorial Hospital, New Taipei \\ City, Taiwan \\ ${ }^{3}$ Department of Internal Medicine, Division of Infection Disease, Far Eastern Memorial Hospital, New Taipei \\ City, Taiwan \\ Email: timothychen921@gmail.com, houtai38@gmail.com
}

Received 29 May 2015; accepted 29 June 2015; published 2 July 2015

Copyright (C) 2015 by authors and Scientific Research Publishing Inc.

This work is licensed under the Creative Commons Attribution International License (CC BY).

http://creativecommons.org/licenses/by/4.0/

(c) (i) Open Access

\begin{abstract}
Neurological manifestations in H1N1 influenza A infection are very rare, especially in adults, and its mechanism of action is still uncertain. Here, we reported the case of a 53-year-old woman with human immunodeficiency virus infection (HIV) who had H1N1 influenza A pneumonia complicated with very rare acute necrotizing encephalitis, although the HIV was under control. With prompt identification and administration of high dosage of dexamethasone, her mental status improved from stupor to clear, with minimal right hemiparesis. Further, brain magnetic resonance image revealed great resolution of mass effect. This dramatic improvement in response to the treatment may improve our understanding of the pathophysiology between H1N1 influenza A infection and acute necrotizing encephalitis.
\end{abstract}

Keywords

H1N1 Influenza A Pneumonia, Acute Necrotizing Encephalitis, Human Immunodeficiency Virus

\section{Introduction}

As H1N1 influenza infection is known to cause severe respiratory complications such as respiratory failure,

How to cite this paper: Chen, K.-C., Gow, C.-H., Yang, C.-J. and Chang, H.-T. (2015) Successful High-Dosage Dexamethasone Treatment of H1N1 Influenza A Pneumonia Complicated with Acute Necrotizing Encephalitis in an HIV-Infected Adult. Case Reports in Clinical Medicine, 4, 245-249. http://dx.doi.org/10.4236/crcm.2015.47048 
acute respiratory distress syndrome (ARDS), and multisystem organ failure; neurological manifestations are rare. Glaser et al. reported that fatal or severe neurological complications of H1N1 influenza A infection were observed in $4 \%$ of patients, and most of them were children. Seizure (2.1\%) was the most frequently observed manifestation, followed by encephalopathy or encephalitis (1.4\%), meningitis $(0.1 \%)$, and Guillain-Barré syndrome (0.05\%) [1]. The national British 2-year surveillance study published in 2014 reported on the emergence of influenza A (H1N1) with associated neurological manifestations in 2009 identified 25 cases, and only 4 adults were positive [2]. Two adults had encephalopathy with movement disorder: one had encephalitis and the other had Guillain-Barré syndrome. All of them had severe neurological deficit and eventually died, but none of them had HIV. Here, we reported a rare neurological complication in HIV-infected woman who had H1N1 influenza A pneumonia.

\section{Case}

A 53-year-old Taiwanese woman with human immunodeficiency virus (HIV) infection (from previous intravenous heroin use) for 10 years was under kivexa and atazanavir treatment to control the disease (HIV viral load undetectable and CD4 cell counts, 757 cells/ $\mu \mathrm{L}$ in November 2013). However, she visited our center in New Taipei City on March 1, 2014, owing to a 4-day history of intermittent fever, general malaise, myalgia, nonproductive cough, dizziness, and progressive dyspnea. She informed us about contacting a family member with the same symptoms, but had no recent travel or exposure to pets or birds. Moreover, she did not receive an influenza vaccination previously. On examination, her blood pressure was 62/34 mm $\mathrm{Hg}$, pulse rate was 128 beats per minute, and ear temperature was $38.4^{\circ} \mathrm{C}$. On auscultation, coarse crackles were noted in the lungs. Neurologic examination revealed a normal mental status, intact cranial nerves, and full strength of muscles. Laboratory test results showed a leukocyte count of $12.96 \times 103$ cells $/ \mathrm{mm}^{3}$ with neutrophil predominance (91\%), an elevated creatine phosphokinase level of $349 \mathrm{IU}$, and mild impairment of renal function (serum creatine level, 1.45 $\mathrm{mg} / \mathrm{dL}$ ). Chest plain film showed bilateral diffuse consolidation. An electrocardiogram showed sinus tachycardia. A nonenhanced brain computed tomographic scan did not show any acute intracranial hemorrhage, territorial infarction, or intracranial mass lesion. On the hospital day 3, dyspnea increased, and emergency intubation of the trachea was performed. Due to acute respiratory distress syndrome (ARDS), she was sedated and paralyzed to facilitate appropriate mechanical ventilation. Bacterial culture and acid-fast smear were negative. Reverse transcription polymerase chain reaction (PCR) for H1N1 influenza A RNA was positive in a nasopharyngeal swab sample. Her ARDS did not improve significantly despite full-course treatment with oseltamivir; therefore, she was subsequently administered the investigational antiviral agent peramivir intravenously after obtaining consent from her family. Fourteen days later, the patient was extubated. However, when the sedation effect faded away, she had an altered mental status and right hemiplegia; thus, the trachea was reintubated later that day. Her brain magnetic resonance imaging (MRI) with T2-weighted and fluid-attenuated inversion recovery sequence revealed abnormal hyperintensities over the right parietal lobe, left parietal-temporal-occipital lobe, and right cerebellar hemisphere. T1 post-gadolinium axial brain MRI sequence revealed leptomeningeal enhancement and multiple areas of microhemorrhage over these regions (Figure 1). The MRI findings were consistent with acute necrotizing encephalitis (ANE). Ceftriaxone, metronidazole, and acyclovir were empirically administered to cover for any of potential brain abscess. We also prescribed $100 \mathrm{mg} /$ day dexamethasone for 3 days for ANE. Mannitol and hypertonic saline were administered intravenously for mass effect caused by brain swelling. Cerebrospinal fluid (CSF) study revealed a white blood cell count level of 0 cells $/ \mu \mathrm{L}$, protein level of $29 \mathrm{mg} / \mathrm{dL}$, and glucose level of $86 \mathrm{mg} / \mathrm{dL}$. Molecular analysis and culture of the CSF were negative for H1N1 influenza A. Three days later, her metal status improved dramatically from stupor to clear. Her consciousness remained clear while we tapered the steroid administration gradually. Sixteen days later, brain MRI with contrast revealed a significant improvement in her necrotizing encephalitis (Figure 2). She was successfully extubated 20 days later and had a normal mental status and right hemiparesis.

\section{Discussion}

To our knowledge, this is the first reported case of H1N1 influenza A-associated ANE in an HIV-infected host. Previous studies have reported that HIV infection did not significantly increase susceptibility to influenza virus infection [3]; however, acquired immunodeficiency syndrome was associated with a higher influenza-related morbidity and mortality, especially in the pre-highly active antiretroviral therapy (HAART) era [4]. Although 


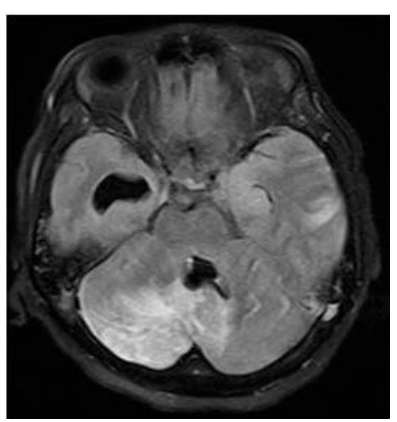

(a)

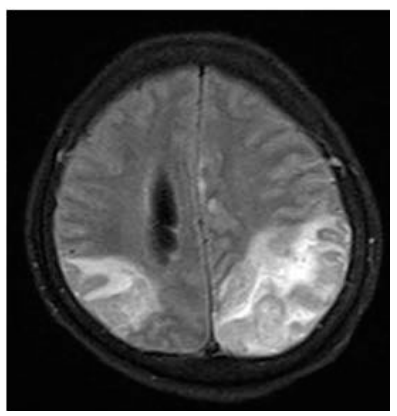

(c)

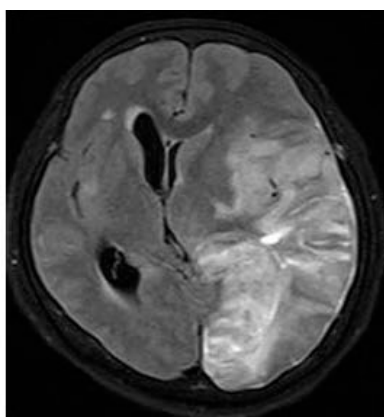

(b)

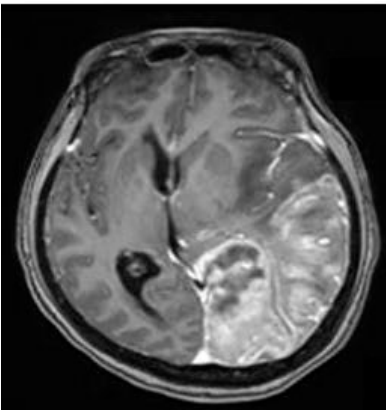

(d)

Figure 1. T2 and fluid attenuated inversion recovery (FLAIR) brain MRI shows hypersignals over the right cerebellar hemisphere (a), left parietal-temporal-occipital area (b), and right parietal lobe (c). These lesions involve both cortical and subcortical area. T1 post-gadolinium axial brain MRI sequence shows meningeal enhancement mainly over the parietaltemporal-occipital area (d), while no ring enhancement is seen. Furthermore, mass effect has caused compression of the left lateral ventricle, resulting in midline shift to the right. Necrotizing encephalitis was impressed in this study.

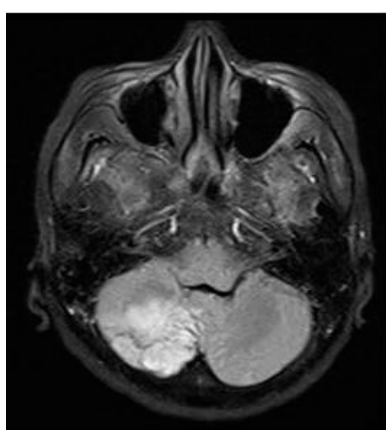

(a)

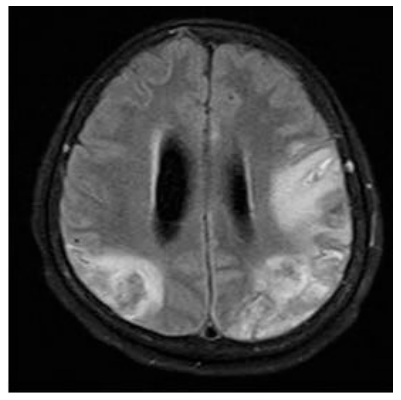

(c)

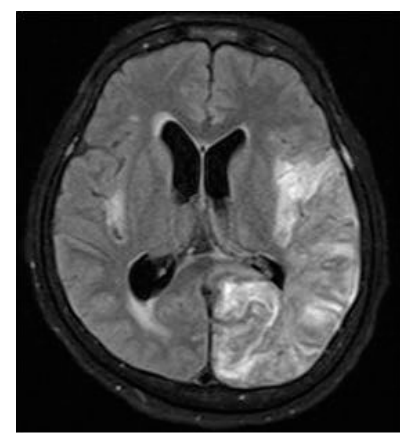

(b)

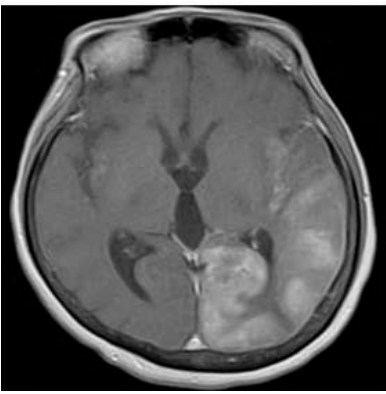

(d)

Figure 2. Sixteen days later, with treatment of mainly antibiotics and steroid, comparison of brain MRI with the same sequence ((a)-(c)) to those of panel 1 revealed that brain swelling with mass effect was greatly resolved, and no more new lesions developed. T1 post-gadolinium axial brain MRI sequence (d) shows less leptomeningeal enhancement. 
data on the duration of influenza shedding in HIV-infected adults are lacking, shedding of the seasonal influenza virus in immunocompromised persons other than HIV hosts may take longer, i.e., from weeks to months [5]. Moreover, the patients easily developed oseltamivir-resistant H1N1 influenza A virus [6].

The role of CD4 cell counts in influenza susceptibility remains unclear. In our case, despite the continuous course of HAART, the CD4 cell counts dramatically declined from 757 to 189 cells/ $\mu \mathrm{L}$ within 4 months, while the HIV viral load was still undetectable. Intriguingly, the patient's neurological manifestation developed after the pneumonia improved, and subsequent brain MRI confirmed typical findings of ANE. It is unclear whether the subsequent ANE occurred due to reduced CD4 cell counts and requires further investigation.

The term ANE was first described by Mizuguchi with the characteristic neuroimaging findings of bilateral, multifocal lesions in the thalami, cerebral and cerebellar deep white matter, brainstem tegmentum, and putamen [7], but its mechanism of action is still uncertain. Some researchers proposed that influenza-like illness preceding neurological manifestations may be attributed to either a cytokine storm or an adaptive, cell-mediated response [8]. Influenza virus has not been previously detected in CSF and brain tissue sample of patients with ANE [9]. Early steroid treatment of ANE was considered beneficial; however, the efficacy of intravenous immunoglobulin was then under investigation [10]. In our patient, the second real time-PCR for influenza RNA from respiratory secretions and cerebrospinal fluid after the occurrence of ANE was negative in tests for the identification of influenza virus. Both her consciousness level and subsequent brain MRI showed great improvement after administration of intravenous dexamethasone (100 mg per day for 3 days). The dramatic improvement after steroid administration may clarify whether the mechanism of ANE is mainly related to immune response. Although most neurological manifestations of influenza infection in adults have a poor outcome, in our case, with prompt identification and subsequent administration of high dosage of steroid, the patient's neurological sequelae were alleviated, and she was successfully weaned from mechanical ventilator afterwards.

\section{Conclusion}

In conclusion, this is the first reported case of an HIV-infected adult with H1N1 influenza A pneumonia with complication of ANE. Although HIV-infected adults are not substantially more susceptible to influenza virus infection, it is unclear whether the low CD4 cell counts here (189 cells/ $\mu \mathrm{L})$ have an influence on H1N1 influenza A virus shedding, causing subsequent ANE, and it requires further investigation. Early detection of ANE especially in sedated patients with H1N1 pneumonia is important, and prompt intravenous steroid administration may alleviate neurological deficits if ANE is highly suspected. Future studies are required to understand the pathophysiology of ANE in patients with H1N1 influenza A infection and identify those in need of an early steroid therapy to improve neurological outcomes.

\section{Acknowledgements}

We would like to thank Dr. Tsai Yi Shin who is a neurosurgeon at Far Eastern Memorial Hospital for his expertise in management of increased intracranial pressure and neuroradiologist Dr. Lai Yan Jun at FEMH for his expertise in interpretation of brain MRI. This research was supported by research grants from the research program of Far Eastern Memorial Hospital, Taiwan (FEMH-2014-C-035).

\section{References}

[1] Glaser, C.A., Winter, K., DuBray, K., Harriman, K., Uyeki, T.M., Sejvar, J., et al. (2012) A Population-Based Study of Neurologic Manifestations of Severe Influenza A(H1N1)pdm09 in California. Clinical Infectious Diseases, 55, 514520. http://dx.doi.org/10.1093/cid/cis454

[2] Goenka, A., Michael, B.D., Ledger, E., Hart, I.J., Absoud, M., Chow, G., et al. (2014) Neurological Manifestations of Influenza Infection in Children and Adults: Results of a National British Surveillance Study. Clinical Infectious Diseases, 58, 775-784. http://dx.doi.org/10.1093/cid/cit922

[3] Cohen, J.P. and Macauley, C. (1989) Susceptibility to Influenza A in HIV-Positive Patients. Journal of the American Medical Association, 261, 245. http://dx.doi.org/10.1001/jama.1989.03420020097023

[4] Neuzil, K.M., Reed, G.W., Mitchel Jr., E.F. and Griffin, M.R. (1999) Influenza-Associated Morbidity and Mortality in Young and Middle-Aged Women. Journal of the American Medical Association, 281, 901-907. http://dx.doi.org/10.1001/jama.281.10.901

[5] Boivin, G., Goyette, N. and Bernatchez, H. (2002) Prolonged Excretion of Amantadine-Resistant Influenza A Virus 
Quasi Species after Cessation of Antiviral Therapy in an Immunocompromised Patient. Clinical Infectious Diseases, 34, E23-E25. http://dx.doi.org/10.1086/338870

[6] Hill-Cawthorne, G.A., Schelenz, S., Lawes, M. and Dervisevic, S. (2010) Oseltamivir-Resistant Pandemic (H1N1) 2009 in Patient with Impaired Immune System. Emerging Infectious Diseases, 16, 1185-1186. http://dx.doi.org/10.3201/eid1607.091579

[7] Mizuguchi, M., Abe, J., Mikkaichi, K., Noma, S., Yoshida, K., Yamanaka, T. and Kamoshita, S. (1995) Acute Necrotising Encephalopathy of Childhood: A New Syndrome Presenting with Multifocal, Symmetric Brain Lesions. Journal of Neurology, Neurosurgery \& Psychiatry, 58, 555-561. http://dx.doi.org/10.1136/jnnp.58.5.555

[8] Akins, P.T., Belko, J., Uyeki, T.M., Axelrod, Y., Lee, K.K. and Silverthorn, J. (2010) H1N1 Encephalitis with Malignant Edema and Review of Neurologic Complications from Influenza. Neurocritical Care, 13, 396-406. http://dx.doi.org/10.1007/s12028-010-9436-0

[9] Mizuguchi, M., Yamanouchi, H., Ichiyama, T. and Shiomi, M. (2007) Acute Encephalopathy Associated with Influenza and Other Viral Infections. Acta Neurologica Scandinavica, 115, 45-56. http://dx.doi.org/10.1111/j.1600-0404.2007.00809.x

[10] Okumura, A., Mizuguchi, M., Kidokoro, H., Tanaka, M., Abe, S., Hosoya, M., et al. (2009) Outcome of Acute Necrotizing Encephalopathy in Relation to Treatment with Corticosteroids and Gammaglobulin. Brain and Development, 31, 221-227. http://dx.doi.org/10.1016/j.braindev.2008.03.005 